\title{
Prevention of gastrointestinal cancer by surveillance endoscopy
}

\author{
René Lambert
}

Received: 11 April 2010 / Accepted: 13 May 2010/Published online: 10 June 2010

(C) European Association for Predictive, Preventive and Personalised Medicine 2010

\begin{abstract}
The classification of the endoscopic appearance of superficial neoplastic lesions of the digestive mucosa aims to evaluate the risk of progression to advanced neoplasia in $3^{\circ}$ (low, intermediate, high) and to predict appropriate treatment and corresponding surveillance. The privileged position of endoscopy results from its double impact on prevention of digestive cancer through reduction in incidence after early detection and eradication of precursors; and through reduction of mortality after detection and treatment of cancer at an early and curable stage. However the efficacy of diagnostic endoscopy still requires improvement and quality control on the following points: (1) technology, with a generalized use of the recently introduced high-resolution endoscopes. (2) diagnosis of poorly visible nonpolypoid precursors: this applies to small depressed lesions and large slightly elevated or sessile serrated and non-serrated precursors, particularly in the proximal colon. (3) treatment and training in therapeutic endoscopy, including the most recent techniques of mucosal resection of nonpolypoid lesions.
\end{abstract}

Keywords Endoscopy · Superficial neoplastic lesion · Early cancer $\cdot$ Mucosectomy $\cdot$ Pathology

\section{Introduction}

Gastroscopy and colonoscopy, as the most generalized endoscopic procedures, are adapted to detect the large majority of digestive cancer in the esophagus, stomach and large bowel. The IARC Globocan database [1], estimates

R. Lambert $(\bowtie)$

I.A.R.C.,

Lyon, France

e-mail: lambert@iarc.fr that cancer located at these three sites account for $24 \%$ of the World cancer mortality in the year 2002. In the aging population of the world, cancer mortality is still expected to increase because of the growing importance of age classes over 60 years. Endoscopic diagnosis of less frequent tumors by enteroscopy or biliopancreatic endoscopy will not be analyzed in this review.

Early detection of cancer at a curable stage and of premalignant precursors may occur in persons complaining from non-specific digestive symptoms, or compliant to a screening intervention. Digestive endoscopy is the gold standard in early detection and has a therapeutic potential. Endoscopic diagnosis underwent a dramatic progress in recent years with high resolution video-endoscopes, optical and electronic magnification and image processing. In relation to this new technology, the strategy of endoscopic diagnosis needs to be revised. This review aims to summarize the morphologic classification of superficial neoplastic lesions in the mucosa of the esophagus, stomach and large bowel and to present a strategy of endoscopic diagnosis based on the prediction of the risk of malignancy in the detected lesions prior to treatment decision.

\section{Burden of digestive cancer}

Tumor sites in the digestive mucosa

In the absence of hiatal hernia, the esophagus joins the stomach at the level of the diaphragmatic pinch and the squamo-columnar epithelial junction, or $\mathrm{Z}$ line is located just above the pinch of the diaphragm. Just above the $Z$ line the stratified squamous epithelium shows characteristic features: palisade vessels and small islands of ectopic cardiac mucosa with a yellowish color. Squamous cell 
cancer is much more common than adenocarcinoma. At the esophagogastric region, confusion may arise between adenocarcinomas developed in the distal esophagus and gastric tumors developed in the proximal stomach. Adenocarcinoma in the stomach includes two sub-sites: proximal (cardia cancer) and distal (non-cardia cancer in the corpus or antrum). Adenocarcinoma in the large bowel is called proximal, or right sided, when located in the cecum, ascending colon, and transverse colon and distal, or left sided, when located in the descending colon, sigmoid and rectum. The intra-luminal fermentation and the bacterial enzymes involved in the generation of mutagenic metabolites are confined to the proximal colon and environmental factors may have a distinct impact on the incidence of proximal and distal tumors.

\section{Parameters in epidemiology}

The burden of digestive cancer (incidence, mortality, survival) is assessed in descriptive epidemiology. New incident cases of cancer are reported in population based cancer registries [2-4]; however in many countries registration is limited to a fraction of the country. Registries files are completed by other sources, including death certificates, medical insurances and other databases $[5,6]$. The annual number of new cases (Incidence) or of deaths from the same disease (Mortality) is reported as a crude rate for 100,000 persons of the population; the crude rate represents the actual burden of cancer at the period selected. The comparison of the risk between two populations differing in their distribution of age classes, requires an adjustment to a modelized world population; the age adjusted or standardized rate (ASR) is established for incidence or mortality per 100,000 persons. Adjustment is also required in the comparison of the risk between two periods in the same country; then the rate is adjusted to the age structure of the population of this country in a fixed year. The global burden of cancer in each country of the world is also evaluated through estimations as in the GLOBOCAN database [1]. Here the worldwide burden of colorectal cancer is estimated in 2002 at 1,023,200 incident new cases and at 529,000 deaths. The respective estimations are 462,000 new cases and 385,900 deaths for esophageal cancer and 904,000 new cases and 700,300 deaths for stomach cancer. Data on survival are extracted from registries with follow-up. The 5-year relative survival (5-y $\mathrm{RS}$ ) describes the percentage of patients alive 5 years after the diagnosis while eliminating other causes of death.

\section{Heritable cancer}

In the esophagus hereditary cancer is exceedingly rare but has been observed in relation with Palmoplantar kerato- derma (Tylosis). In the stomach hereditary cancer is also rare and caused by a germline mutation of the regulatory gene (16q) encoding E-cadherin. Gastric cancer (intestinal type of Lauren) may occur in other genetic syndromes: hereditary non-polyposis colon cancer and hereditary gastrointestinal polyposis (FAP and Peutz-Jeghers syndrome). In the colon hereditary cancer, represents near to $5 \%$ of all cases; the two major categories are the FAP and the HNPCC syndromes.

\section{Temporal variations in incidence}

Temporal variations of the age adjusted incidence are caused either by environmental factors or by a policy of prevention and early detection.

Squamous cell cancer in esophagus The ASR incidence/ 100,000 (adjusted to world population) is low, in the range 5 to 15 , in most Western countries. The incidence is higher in regions with a low socio-economic status and nutritional deficiencies in Asia [7, 8], Northern India and Western China and in South Africa (Transkei). The ASR incidence/ 100,000 (adjusted to world population) for the period 1993-97 in Cixian county adjacent to Linxian county in China is 183.3 in men and 123.1 in women [2]. In Western countries where most cases develop in relation to alcohol and tobacco consumption; a decreased in incidence occurs over the past 20 years in men, in relation to decreased consumption of alcohols. In regions of Asia, Africa, at high risk for esophageal cancer, common factors in diet and environment cause a chronic inflammation of the mucosa with esophagitis and cancer; recent surveys indicate that incidence decreases, perhaps in relation with improved socio-economic status. The 5y-RS is low; as an example the median figure is only at $12.3 \%$, in cancer registries of the European Union during the period 1995-99 [5].

Adenocarcinoma in esophagus The tumor develops in a segment of columnar metaplasia called a Barrett esophagus [9-13]. The risk of malignancy in columnar metaplasia is evaluated as one case for 200 years-patient. In registries without histology subgroups, the incidence of adenocarcinoma is joined to that of squamous cell cancer. In period 1998-2002, the respective proportions of adenocarcinoma are given by registries with histology subgroups for esophageal cancer [2]. The figures were high in the USA and northen Europe: $59.5 \%$ for the white non Hispanic in the USA [4], $44.9 \%$ in Denmark, $53.0 \%$ in Netherlands. Much lower figures occurred in other countries: $4.1 \%$ in Japan [3], 3.9\% in Brazil (Sao Paulo registry). There is a contrast between the increasing incidence of adenocarcinoma in the esophagus with the stable or decreasing incidence of adenocarcinoma at the cardia during the last quarter of 
the XXth century [11]. Several studies have shown that an increased risk of neoplasia in columnar neoplasia is linked to Caucasian ethnicity, male sex, old age, alcohol consumption, continuous smoking, dietary deficiency in fruit and vegetable and a long history of reflux symptoms.

Adenocarcinoma in stomach This tumor is still the second most frequent cause of death from cancer. The localization in the proximal stomach or cardia, is less frequent than in the distal stomach, which accounts for more than $80 \%$ of cases [14]. The risk of gastric cancer in men is about twice that of women. Areas of high incidence occur in Eastern Asia (Japan, Korea, China), Western South America and Eastern Europe [12-14]. In 1998-2002 period the ASR incidence/100,000 (adjusted to world population) [2] is 7.2 in men and 3.4 in women in the USA [4]; the respective figures are 7.1 and 3.2 in Denmark; 51.3 and 19.8 in Japan [3], 43.1 and 16.0 in Chile (registry of the Valdivia province). The causal factor is chronic atrophic gastritis provoked by $\mathrm{H}$. Pylori. The worldwide declining incidence of distal stomach cancer is attributed to environmental factors in diet and control of $\mathrm{H}$. pylori infection. In Japan [3], the incidence decreased during the period $1963-89$ by $47 \%$ in men and $43 \%$ in women. In Western countries where most cases are detected at an advanced stage the 5-y RS is still low in 2002-2004 with a median value at $24.9 \%$ in European Countries [5] and 25\% in the USA [4]. The higher proportion of tumors detected at the localized stage in Japan explains a higher survival: $47 \%$ in the Osaka registry in the period 1987-89.

Adenocarcinoma in colon and rectum This tumor is the second most frequent cancer. The incidence is high in countries of North America, Europe, Australia and in Japan, and still low in developing countries of Africa and in central Asia. In 1998-2002 the ASR incidence $/ 100,000$ (adjusted to world population) [2] is 38.6 in men and 28.3 in women in the USA [4]; the respective values are 37.4 and 21.7 in Japan [3], and 39.3 and 29.8 in Denmark. The Ocidental style of life with a higher alimentary intake, excess weight shown in the variation of the BMI and decrease in physical activity explains the increased incidence. The generalization of this life style occurs in emerging countries and also in Japan where the ASR incidence/100,000 persons (adjusted to Japanese population) passed from 10,9 in 1975 to 43,8 in 1999 in men [6]. Noteworthy the development of early endoscopic detection and treatment of precursors in developed countries explains the temporal decrease in incidence, while survival is increasing. In the USA [4] during the period 1975-2002, the 5-y RS progressed from $50.2 \%$ to $65.4 \%$ in men; and from $52.3 \%$ to $66.3 \%$ in women. In Japan the 5 -y RS progressed from $37.1 \%$ in $1962-66$ to $71.7 \%$ in $1992-96$ in men; the respective values in women were $32.8 \%$ and $72.1 \%$ [6]. In registries from the European Union [5] the 5y RS (both sexes) of colorectal cancer slightly progressed from $54 \%$ in the period $1995-1999$ to $56.8 \%$ in the period 2000-2002.

Precursors and premalignant lesions

A precursor is the temporal ancestor of a neoplastic premalignant lesion; this definition applies to chronic inflammation of the digestive mucosa as chronic esophagitis in some regions of Asia and Africa, columnar metaplasia in the esophagus, chronic atrophic gastritis in the stomach, chronic inflammation of the colonic mucosa in inflammatory bowel disease. However a neoplastic lesion will never develop in the majority of precursors and the place where it may arise within a diffuse alteration of the mucosa cannot be determined. The assessment of a precursor as a "preneoplastic" lesion depends upon the presence of reliable histological criteria and of molecular markers: the most common molecular alterations are mutations in the suppressor gene TP53 (17p13), encoding p53, inactivation of $P 16 / C D K N 2 A$ (9p22), encoding p16, amplification of CCND1, encoding Cyclin D1, and overexpression of proto-oncogenes such as the EGF receptor or c-Myc. Neoplastic lesions develop in a normal epithelium, or in a mucosa diffusely altered by a precursor and preneoplastic condition. Neoplastic lesions are circurmscribed alterations with cell atypia, architectural disorganization, and evidence of hyperproliferation, which carry a significant risk of progression to malignancy.

Squamous cell cancer in esophagus Reflux esophagitis in the Western World is not a precursor of esophageal cancer and patients with gastroesophageal reflux and chronic erosive esophagitis are not at increased risk for cancer [1]. However chronic erosive esophagitis in alcoholic patients is a preneoplastic condition. In Asia and Africa the role of non erosive chronic esophagitis as a preneoplastic condition [11, 12] has been suggested (but still debated) owing its very high prevalence in the regions with high prevalence of esophageal cancer (Henan, China and Transkei, South Africa).

Adenocarcinoma in esophagus Columnar metaplasia in the esophagus is a preneoplastic condition [10-12]. There is still debate with respect to the requirement of intestinal metaplasia to the status of preneoplastic condition. Based on endoscopic series conducted in a random sample of population, the prevalence of columnar metaplasia in the adult population is estimated at $1.6 \%$ in a study conducted in Sweden [13]. In Asian populations the prevalence is 
lower. The risk of cancer increases with the length of the segment, but short segments deserve as much attention because they are more frequent. The surgical correction of reflux does not suppress the risk of cancer.

Adenocarcinoma in stomach Chronic atrophic gastritis in the mucosa infected by $\mathrm{H}$. pylori is a preneoplastic condition $[15,16]$. The sequence leading from chronic gastritis to gastric cancer has been described by Correa [15] and occurs in most cases of cancer. The progression to cancer is slow low grade intraepithelial neoplasia and rapid in a few months high grade intraepithelial neoplasia. The Lauren classification of gastric adenocarcinoma is based on 2 categories: a "intestinal" type with recognizable glands and a "diffuse" type without cohesion between the malignant cells. Adenocarcinoma with a tubular, papillary, mucinous or signet ring cell pattern is classified as well, moderately, poorly differentiated.

Adenocarcinoma in colon and rectum Chronic inflammation plays no role in the development of most neoplastic lesions of the colorectal mucosa. However the chronic inflammatory changes occurring in Ulcerative Colitis and in Crohn's disease can be considered as preneoplastic precursors. Until recently, it was assumed that all colorectal neoplastic lesions developed through the single sequence of polypoid adenoma cancer with mutations involving APC inactivation, KRAS mutation and TP53 inactivation in the sequence. New molecular approaches, based on DNA sequencing with polymerase chain reaction (PCR), have demonstrated distinct colon cancer classes and genomic profiles in nonpolypoid adenomas [17]. Furthermore serrated lesions provide a common pathway between neoplastic and non-neoplastic lesions and a fraction of hyperplastic polyps are preneoplastic lesions. As in the stomach colorectal adenocarcinoma is classified as well, moderately or poorly differentiated.

\section{Superficial neoplastic lesions}

The premalignant, or malignant, neoplastic lesions of the digestive mucosa are called superficial when the endoscopic appearance suggests that invasion is limited to the mucosa (m) or the submucosa (sm). They are classified as type 0 in Japan and in the Paris classification [18], while advanced and non superficial cancer is classified in types 1 to 5 . The gross morphology of type 0 lesions is described in subtypes: - type I polypoid, with variants pediculated or sessile (Ip, Is), - type II nonpolypoid, with variants slightly elevated, flat or slightly depressed (IIa, IIb, IIc) -type III ulcerated. The proportion of nonpolypoid lesions is estimated in various studies from Japan at $84 \%$ in esophagus, $95 \%$ in the stomach and $49 \%$ in the colon [18]. The morphology has a prognostic value, irrespective of the size of the lesion and the depressed (0-IIc) type, in spite of being rare plays a significant role as precursor of advanced cancer.

In type 0 lesions, pathologists stratify the risk of cancer according to the Vienna classification [19]. Groups 1 (negative for neoplasia) and 2 (indefinite for neoplasia) correspond to a preneoplastic condition. Groups 3 (low grade intraepithelial neoplasia) and 4 (high grade non invasive or invasive intraepithelial neoplasia) correspond to a neoplastic lesion. Group 5 corresponds to cancer with invasion of the submucosa. In the columnar type of epithelium high grade premalignant non invasive lesions are included as well as intramucosal invasive lesions in the same Group 4 of the Vienna classification. In the squamous epithelium intramucosal cancer is classified as intraepithelial, microinvasive or intramucosal.

The terminology "early cancer" is also used for a malignant superficial neoplastic lesion and assessment of invasion in the submucosa is critical for the risk of nodal metastases. A micrometric measure of the depth of invasion from the lower limit of the muscularis-mucosae helps to control the legitimacy of localized treatment by endoscopy. The empirical cut-off limit of invasion is $200 \mu \mathrm{m}$ in the squamous stratified epithelium of the esophagus, $500 \mu \mathrm{m}$ in the columnar epithelium of the stomach and $1,000 \mu \mathrm{m}$ in the columnar epithelium of the colon. In addition to depth of invasion, the presence of qualitative indices of poor prognosis (tumor grade, images of vascular invasion, tumor budding) is explored.

\section{Endoscopic diagnosis}

With high resolution electronic video-endoscopes coupled to HDTV standard, image processing increases the contrast of the mucosal surface as in the Narrow Band Imaging (NBI) or FICE techniques [20-26]. The architecture (pits and ridges) of the normal mucosa is now described in magnification up to $\times 150$ with the optical and electronic zoom, including the description of epithelial types as shown in Barrett's esophagus [27] As an approach to in vivo cytology or histology, magnification up to $\times 1,000$ is reached with the endo-cytoscopy probe or with Confocal Laser Endomicroscopy [28-30]. The improved technology is not a substitute to a careful exploration of the mucosa during endoscopy. The analysis of the lesion is even more important than before because more abnormalities are being visible. Endoscopic diagnosis is not a single step procedure: - First is the detection of an area with an abnormal apperance - Second is the characterization of 
the target area - Third is treatment decision, based on a pragmatic classification [31].

\section{Detection}

Small lesions can be masked by any solid and liquid matter persisting at the surface of the mucosa. The exigence of complete cleanliness of the digestive lumen applies to the large bowel after a cathartic preparation but also to the upper digestive tract where all particles inside the stomach should be removed with a jet of water and aspiration. During upper GI endoscopy or colonoscopy the surface of the mucosa is explored sector by sector, including blind zones. Abnormal areas are detected in standard vision without image processing and chromoscopy, on any of the following elements: - obvious elevation or depression; mucosal discoloration-interruption of the course of superficial capillaries. Polypoid, sessile or pedunculated, neoplastic lesions are conspicuous, even when small, but can be missed in blind areas. Nonpolypoid lesions, even large, can be missed when the operator lacks cognitive training for a slight change in color of the mucosa. Special attention is required in the exploration of the proximal colon, where poorly visible lesions are often covered by bile stained mucus. Then, the morphology and limits of the lesion are described with chromoscopy. The non absorbed dye, a solution of indigocarmine $(0.5 \%)$, can be used everywhere; other absorbable dyes have specific indications: the iodine-potassium iodide (1.5 to $2 \%$ ), the acetic acid (3 to $5 \%$ ), methylene blue $(0.5 \%)$ in the stomach, crystal violet $(0.2 \%)$ in the colon. The size of the lesion can be measured with a gauge, or classified as diminutive (up to $5 \mathrm{~mm})$, small or intermediate $(6-9 \mathrm{~mm})$ or large $(10 \mathrm{~mm}$ and more) and the morphology is classified in the subtypes of the Paris classification.

\section{Characterization}

Characterization classifies the lesion prior to pathology, in introduction to treatment decision based on the evaluation of the risk of malignancy in lesions with a neoplastic appearance [3-34]. Therefore the rules of the analysis are the same for premalignant precursors and for superficial cancer. The histopathology prediction is based at first on gross morphology (Figures 1, 2, 3, 4 and 5): in non depressed lesions (type 0-I, IIa, IIb) the risk of invasive malignancy increases with the size. In depressed lesions (type 0-IIc) the risk occurs even if they are small and less than $6 \mathrm{~mm}$ in diameter. Then the surface microarchitecture is analyzed with magnification, combined with dye chromo-endoscopy and the network of superficial capillaries is analyzed in transparency. The NBI technique has recently confirmed its efficacy in this analysis.
The gross morphology

In the esophagus the usual morphology of superficial squamous cell cancer is flat or depressed; in a metanalysis of 143 centres conducted in Japan the distribution in the subtypes was as follows: 0 -I in $2.2 \%$, 0 -IIa in $12.6 \%, 0$-IIb in $31.4 \%$, 0 -IIc in $53 \%, 0$-III in less than $1 \%$ [18]. In the columnar lined esophagus superficial premalignant lesions and adenocarcinomas are suspected on the basis of slight surface irregularities, spots with color change, or variations in the network of superficial vessels. Pediculated polypoid (-0-Ip) lesions are rare, esophageal ulcers are associated to fibrosis and strictures, mixed types of tumor associate sessile nodules, often multiple and a depressed area.

In the stomach superficial premalignant lesions and adenocarcinomas appears ad discolored (pale or red) areas in the mucosa, with minimal alterations of the surface after chromoscopy. Most neoplastic lesions are flat or depressed (type 0-II c). The large majority of polypoid lesions corresponds to non-neoplastic polyps (fundic cystic polyps and hyperplastic polyp). Premalignant gastric lesions are described as flat adenomas or depressed adenomas. Malignant lesions are described as early cancer. In Japan, during National Screening Campaigns, around $70 \%$ of type 0 gastric cancer show this nonpolypoid depressed morphology.

In the colon, the morphology of adenomas is often polypoid, pedunculated or sessile, and classified in subtypes 0 -Ip and 0-Is of the Paris classification. The direction of growth of those adenomas is upwards, increasing in volume more than in depth across the colonic wall. The majority of polypoid adenomas are small and adenomas larger than $10 \mathrm{~mm}$ are often called "advanced" as well as those showing at least a $25 \%$ villous architecture, or high-grade intraepithelial neoplasia. The majority of nonpolypoid and nondepressed adenomas are classified in subtype 0-IIa, just as the majority of non-neoplastic hyperplastic polyps [34]. Large adenomas with a growth pattern more lateral than vertical and a global nonpolypoid morphology are called Laterally spreading tumors (LST) and are classified in two groups: Granular with an homogeneous or nodular surface (0-IIa or IIa + Is) and non granular with an even surface often depressed, (0-IIa or IIa + IIc). Depressed adenomas (subtype 0 -IIc) account for only $5 \%$ of superficial neoplastic lesions but show a higher proportion of submucosal invasion than other sub-types. Depressed 0-IIc lesions differ from the socalled "flat depressed adenomas" by the abrupt edge of the central depression. In Japan, submucosal invasion occurred in $35.9 \%$ of superficial depressed lesions in the very large series $(n=25,862)$ collected in the Akita and Yokohama Hospitals in Japan and in $27 \%$ of the series $(n=12811)$ collected in the Hiroshima University Hospital [18, 34]. Sessile serrated lesions are large, with a size over $10 \mathrm{~mm}$ in diameter, and follow a predominantly lateral growth pattern. 
Fig. 1 Superficial adenocarcinoma of stomach at angulus, staged T1,N0. At left, standard vision: a flat area with a reddened appearance. At right, indigocarmine chromoscopy: a $10 \mathrm{~mm}$ long depressed lesion, reddened in depth with clear borders; classified 0-IIc
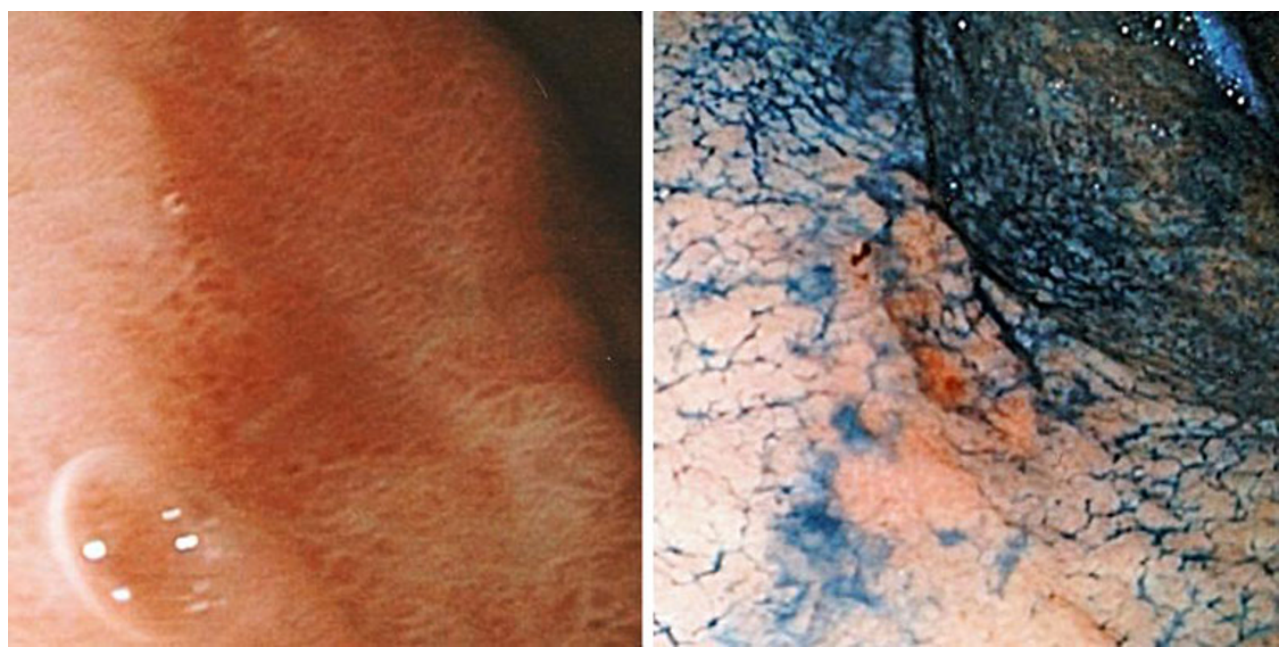

Their general morphology is nonpolypoid ( 0 -IIa, or IIa + Is). The surface of the lesion is homogenous and often covered with a yellow mucous layer; sessile serrated lesions have a significant risk of progression to serrated adenoma. Serrated adenomas offer a polypoid or nonpolypoid morphology, corresponding to the subtypes 0 -Ip, Is or IIa.

\section{The microarchitecture in surface}

The surface microarchitecture of the epithelium (depressions and ridges) is visible when using NBI or chromoscopy with the optical zoom or the objective macro.

In the squamous epithelium of the esophagus the surface of the normal epithelium has a smooth appearance with a clear pink color. In areas with neoplasia the spontaneous colour is changed before using the chromoscopy with the the iodinepotassium iodide solution [35]. In the irregular surface the most patent change is in the regression of the multiple red dots corresponding to the intrapapillary capillary loops therefore in exploring the microvascular network [25].
In the columnar lined esophagus, characters highly suggestive ofneoplasia include an abrupt change in the average size of the epithelial crests (from large to small) with irregular and distorted pattern, the presence of areas with an amorphous surface and abnormal corkscrew microvessels [21].

In the stomach the most frequent aspect of the surface of early gastric cancer is gyrus and villous pattern in place of round pits; the epithelial crests are either regular or irregular; an amorphous pattern of surface is suggestive of submucosal invasion [24].

In the colon the surface microarchitecture of epithelial pits, grooves and crests is classified in the subtypes of pit pattern mostly described in Japan [20, 23, 36-41]: Type I with small and regular pit openings suggesting a normal mucosa. Type II with large and regular stellar pit openings suggesting a non-neoplastic, hyperplastic lesion. Types IIIL, IIIS, IV, IV, Vi, VN with sinuous short, long, or branched crests, suggesting neoplasia and progression from a low- to a high-risk of malignancy. In adenomas, less than

Fig. 2 Advanced adenocarcinoma of stomach at angulus,Staged T3,N1. At left, standard vision: a flat reddened area surrounded by a swollen margin. At right, indigocarmine chromoscopy: a depression surrounded by multiple nodules, $15 \mathrm{~mm}$ in diameter. Not classified as a superficial lesion
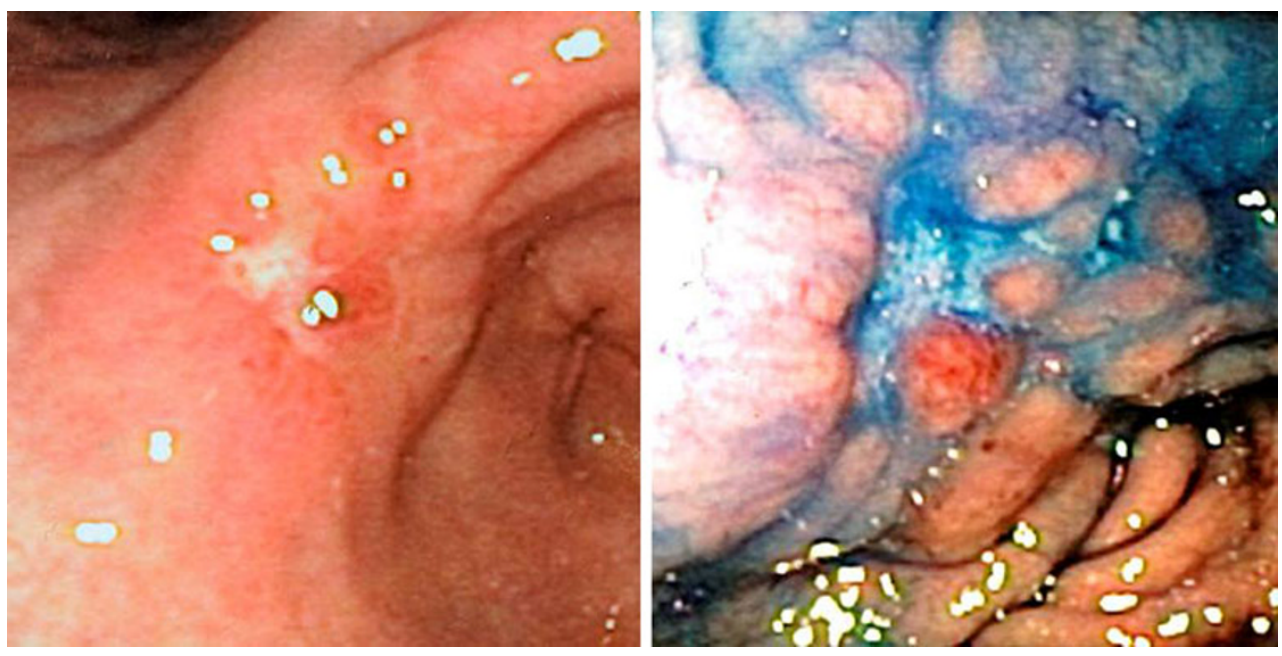
Fig. 3 Hyperplastic sessile polyp in the colon. At left, standard vision: a sessile lesion with a small nodule at on extremity. At right, indigocarmine chromoscopy: the lesion is $10 \mathrm{~mm}$ in length, with a pit pattern type II with large openings; classified 0-IIa + Is
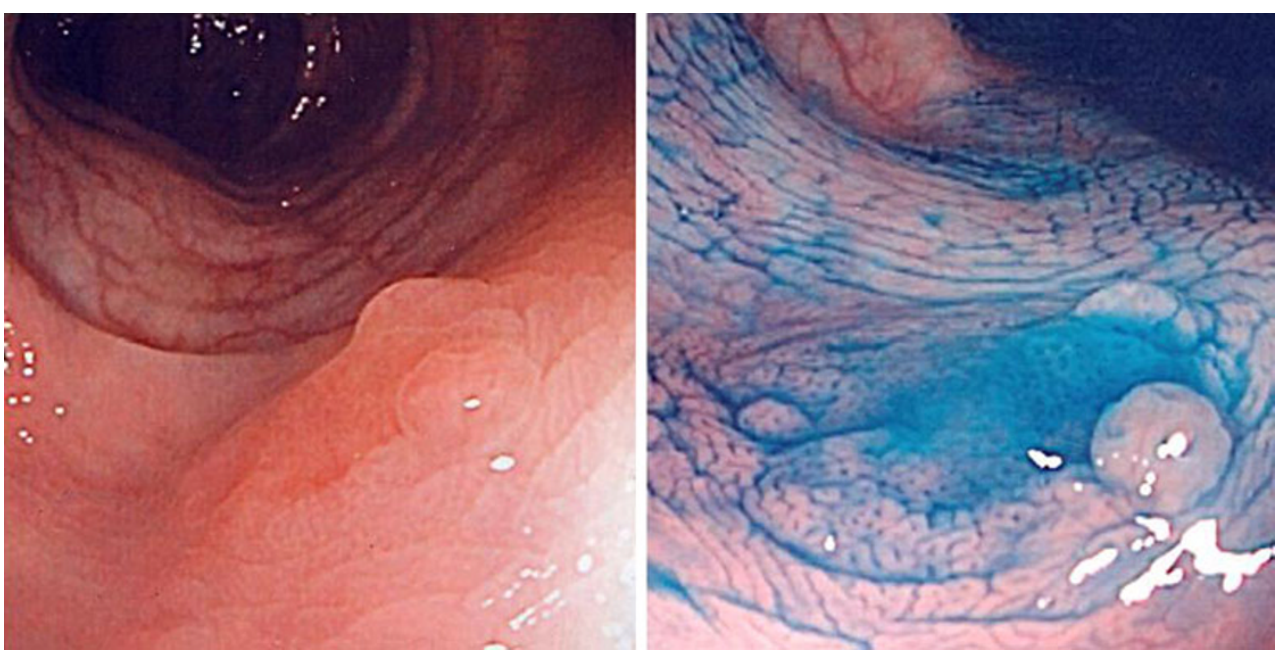

$10 \mathrm{~mm}$ in diameter a pit pattern in categories IIIL or IV confirms a relatively low potential of evolution to adenocarcinoma. The degree of irregularity of the pit pattern $\mathrm{Vi}$ predicts the depth of invasion in the submucosa below or above the limit of $1,000 \mu \mathrm{m}$. The terminology de novo cancer corresponds to small lesions with a flat or depressed nonpolypoid morphology, and a pit pattern classified in categories $\mathrm{Vi}$ to $\mathrm{VN}$. Invasion in the sub-mucosa is frequent, as is its progression to ulcerated advanced cancer. In serrated adenomas, which frequently progress to serrated carcinoma, the surface pit pattern varies from type II to type IIIL in small lesions, to type IV in large serrated adenomas and to type Vi or VN in serrated carcinomas.

\section{The microvascular network}

Subepithelial capillaries are explored in transparency (no chromoscopy) during endoscopy, or with NBI where small vessels are clearly contrasted in dark green. In the normal mucosa the subepithelial capillaries reproduce the architecture of the surface and vary with the site.

In the squamous epithelium of the esophagus intrapapillary capillary loops (IPCL) show a hair-clip appearance. In neoplastic lesions the vascular pattern varies from IPCL to a punctuated pattern with tumoral neoangiogenesis and development of vessels with an irregular and larger diameter, predictive of depth of invasion [42].

In the columnar lined esophagus the microvascular network at the surface of neoplastic areas has some relevance to the depth of invasion. Slight vascular alterations suggest low grade neoplasia and severe vascular alterations suggest high grade non-invasive or invasive neoplasia [43].

In the stomach subepithelial capillaries form a honeycomb network around the neck of gastric pits in the oxyntic mucosa and present as coiled subepithelial capillaries in the antral mucosa. The surface of intramucosal cancer shows abnormal vessels with a mesh, coil or corkscrew pattern according to the differentiation of the tumor $[24,44]$.
Fig. 4 Laterally spreading lesion (LST) in the colon with high grade intraepithelial neoplasia. At left, standard vision: a protruding nodule $6 \mathrm{~mm}$ in diameter surrounded by a slightly surelevated area with uncertain limits. At right, indigocarmine chromoscopy: the lesion is over $25 \mathrm{~mm}$ in length. Classified 0 IIa + Is and as a nodular mixed type of granular LST
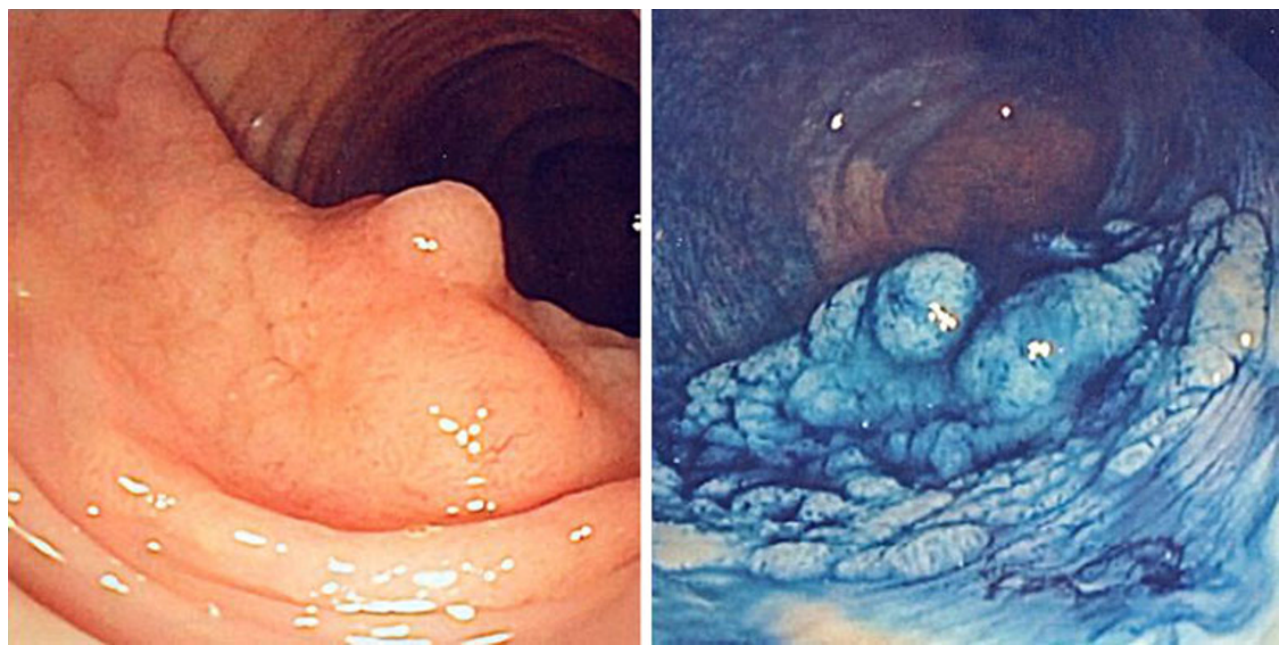
Fig. 5 Laterally spreading lesion (LST) in the colon with high grade intraepithelial neoplasia. At left, standard vision: a sessile lesion $20 \mathrm{~mm}$ in diameter with sharp limits and a central depression. At right, indigocarmine chromoscopy and magnification: epithelial crests show a villous pattern. Classified as 0 -IIa and as a nodular type of granular LST
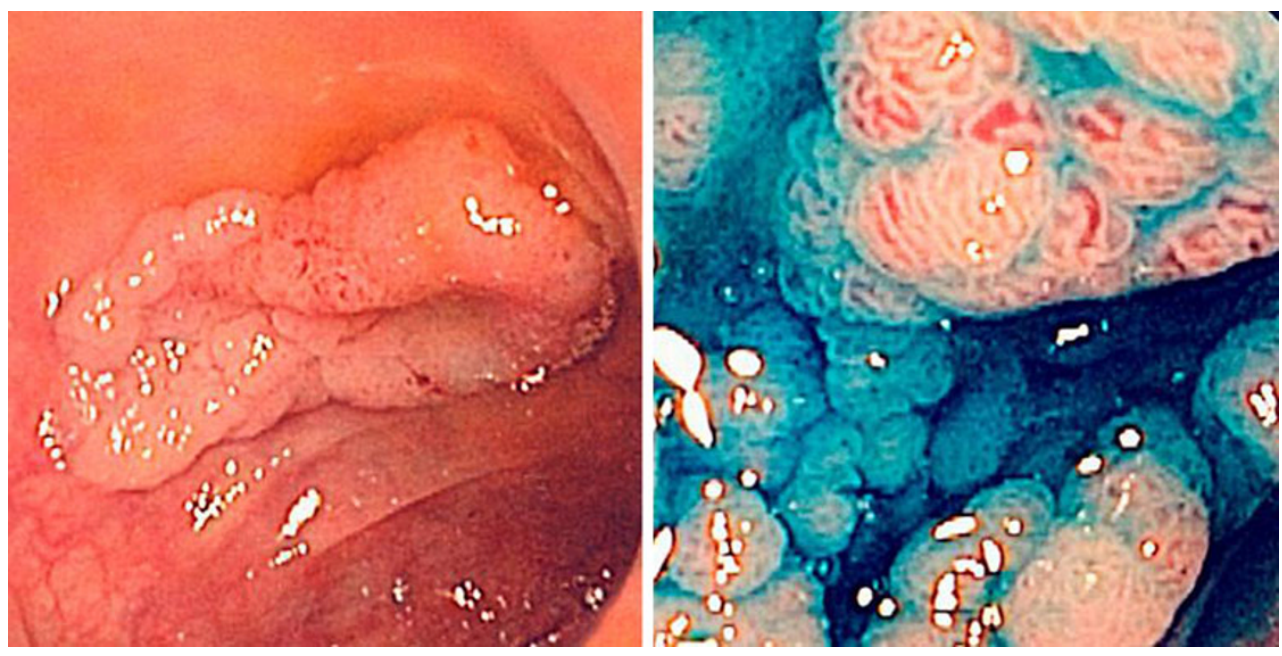

In the colon capillaries ensure a hexagonal network around the pits. The successive categories of pit pattern-FaintNetwork-Dense-Irregular-Sparse - correspond to the transition from non-neoplastic to malignant neoplasia [45].

Pragmatic classification and treatment decision

\section{Application to columnar epithelium}

The next step after endoscopic detection of a superficial lesion developed in the columnar epithelium in the esophagus with columnar metaplasia, in the stomach or the large bowel, is treatment decision. The options are as follows:

1 - no treatment.

2 - endoscopic mucosal resection or EMR which is limited by its inability to achieve en bloc resection for lesions larger than $25 \mathrm{~mm}$. This limit is overcome in the new technique of endoscopic submucosal dissection (ESD); however the procedure requires more time and technical skill, and needs to be standardized for application in the colon.

3 - surgery.

The legitimacy of treatment decision is confirmed by the results of histopathology, using the Vienna classification of neoplasia in the columnar epithelium into five categories of which categories 1 and 2 apply to non-neoplastic lesions. A strategy of endoscopic diagnosis with prediction of histology based upon high resolution and magnification combined with chromoscopy or NBI technique, is accessible to most endoscopy units. The following scale of risk of lymph node invasion and progression to advanced cancer can be proposed:

No risk of lymph node invasion This corresponds to categories 1 and 2 in Vienna classification and applies to normal epithelium, inflammation and small hyperplastic polyps in the colon less than $10 \mathrm{~mm}$ in diameter, which are considered as non-neoplastic lesions. Treatment decision is based on size: small hyperplastic polyps are not treated, polyps larger than $10 \mathrm{~mm}$ and sessile serrated lesions are resected. Surveillance is optional in absence of treatment.

Low risk of lymph node invasion This corresponds to categories 3 and 4-1 to 4-4 of the Vienna classification and applies to low-grade intraepithelial neoplasia and noninvasive high-grade intraepithelial neoplasia, In the stomach and colon a well-differentiated intra-mucosal carcinoma with low-grade cell atypia is also included in this category because the risk of lymph node metastasis is considered to be near to nil. Endoscopic resection of the lesion is proposed. Follow-up is required, with surveillance intervals adapted according to the histopathology.

Intermediate risk of lymph node invasion This corresponds to category 4-4 in the Vienna classification and partly to category 5. It involves three types of lesions: differentiated intra-mucosal carcinoma with high-grade cellular atypia; poorly differentiated intra-mucosal carcinoma with signet ring cells; - well differentiated sub-mucosal carcinoma with depth of invasion in the submucosa less than $500 \mu \mathrm{m}$ in stomach and over $1,000 \mu \mathrm{m}$ in colon and with low-grade cell atypia. The risk of lymph node metastasis is estimated from 1 to $4.8 \%$. There is an option between endoscopic or surgical resection. Surveillance at short intervals is required after treatment. Surgery is recommended if the histopathology reveals a high-risk lesion.

High risk of lymph node invasion This corresponds to category 5 in the Vienna classification including two types of lesions: - sub-mucosal poorly differentiated carcinoma; well-differentiated sub-mucosal carcinoma with high-grade cell atypia or invasion deeper than $500 \mu \mathrm{m}$ in stomach or $1,000 \mu \mathrm{m}$. Surgical treatment with/without neo-adjuvant therapy is required as well as surveillance after treatment. 


\section{Application to squamous epithelium in the esophagus}

A specific classification taking in account the structure of the squamous epithelium in the esophagus is justified: in the mucosa the risk of lymph node invasion is not nil when there is invasion of the lamina propria below the epithelial layer. Intramucosal squamous cell cancer is classified with respect to the depth of invasion as intraepithelial, microinvasive, or intramucosal: when there is deep invasion of the submucosa, a superficial carcinoma is classified as advanced cancer. Concerning lymph node metastases - the risk is still extremely low (less than $2 \%$ ) in superficial cancer classified $\mathrm{m} 1$ or $\mathrm{m} 2$ which correspond to intraepithelial and to microinvasive cancer; endoscopic treatment is recommended - the level of the risk is intermediate and already sizable (just under 20\%) in cancer classified $\mathrm{m} 3$ and the choice between endoscopic treatment or surgery depends on the health status of the patient. When there is sub-mucosal invasion, the risk is moderate is sml cancer but extremely high ( $40 \%)$ is $\mathrm{sm} 2$ or $\mathrm{sm} 3$ cancer. Actually endoscopic resection is still recommended if the invasion is less than $200 \mu$; over this limit the indication is surgery. The endoscopic treatment of a superficial neoplastic lesion in the squamous stratified epithelium of the esophagus is legitimate in approximately $20 \%$ of type 0 -IIa lesions, $39 \%$ of type 0 -IIc lesions and $69 \%$ of type 0 -IIb lesions.

\section{Digestive endoscopy in screening}

Endoscopic surveillance in the prevention of digestive cancer

Surveillance, refers to endoscopy scheduled at regular intervals from 2 to 5 years, independently of symptoms, in those patients who do not have cancer, but who are at increased risk. For the esophagus surveillance is justified in persons having high consumption of alcohol and tobacco, or antecedents of cancer in the upper aero-digestive tract, in persons having columnar metaplasia in the esophagus in the male sex with a prolonged symptomatic history of GERD, continuous smoking, in presence of a peptic stricture or a esophageal ulcer at endoscopy. For the stomach the most frequent reason for surveillance is the presence of chronic acquired atrophic gastritis in the postoperative stomach and in pernicious anemia. For the colon this applies to persons having a genetic predisposition to colorectal cancer in their family without germline mutation type FAP or HNPCC.

\section{Screening}

Secondary prevention of digestive cancer aims to early detection at a curable stage in asymptomatic persons. Early detection of confirmed cancer reduces cancer mortality without impact on incidence. Early detection and treatment of precancerous lesions has in addition an impact on incidence. Organized population based (mass screening) and individual screening (opportunistic screening) are complementary and variable situations occur in individual detection. Population based screening associates a relatively simple filter test, and a diagnostic test in persons with a positive test. In the digestive tract upper GI endoscopy or colonoscopy are the gold standard diagnostic tests. For individual opportunistic screening, endoscopy is the primary procedure. Limiting factors in the benefit of early detection, and treatment of neoplastic lesions include-the degree of compliance to the detection procedure or to recall for treatment in persons positive to the test-the overdetection and over-treatment of lesions with a very low risk of progression to malignancy, - the miss-rate for small, but evolutive lesions, often responsible of interval cancers after a negative exploration.

For esophageal squamous cell cancer, opportunistic endoscopic screening may be proposed to persons having high consumption of alcohol and tobacco, or antecedents of cancer in the upper aero-digestive tract. In China, in areas of high risk, population based screening campaigns based on cytology smears and also on upper GI endoscopy have been conducted.

For adenocarcinoma in the columnar lined esophagus, the incidence is too low to justify mass screening protocols, and there is only limited evidence on the benefit of screening persons with unsedated transnasal endoscopy and capsule endoscopy.

For adenocarcinoma in the stomach, gastroscopy in spite of its efficacy, has a significant miss rate for early cancer evaluated at $19 \%$ in a Japanese study. The consensus view is that screening for gastric cancer does not deserve a public Health Policy outside of Japan. In this country mass screening, based on photofluorography, as a filter test reaches a large proportion of the population and the percentage of ever-screened subjects in Japan is estimated around $60 \%$. The serum pepsinogen test, has shown efficacy as a filter test alternative to photofluorography in screening protocols. However opportunistic screening with upper GI endoscopy is still the major source of detection for early gastric cancer.

Screening for colorectal cancer is now frequently supported by the Health Policies of most developed countries. It is based on a simple filter test, the fecal occult blood test (guaiac or immunological) followed by colonoscopy in persons positive to the test. Screening with primary colonoscopy is also developing rapidly [46]; the role of nonpolypoid lesions is now acknowledged in Western countries [47]. In spite of its efficacy [48] the technique of colonoscopy still deserves to be improved and 
the proximal colon (cecum and ascending colon) is the preferential site of large but poorly visible neoplastic lesions easily missed during a screening colonoscopy. A population-based, case-control study conducted in Ontario, Canada [49], confirmed that there was no protection afforded by a previous colonoscopy for proximal cancer. There was a sizable reduction in the risk of distal colorectal cancer but no impact on death from right-sided CRC. This result was confirmed by a further similar study conducted in Germany. Interval cancers revealed a few years after a negative colonoscopy often correspond to missed lesions during the initial exploration.

(Illustrations with courtesy from Annick Chavaillon)

\section{References}

1. Ferlay F, Bray P, Pisani et al. GLOBOCAN 2002 cancer incidence, mortality and prevalence worldwide. IARC CancerBase No. 5, version 2.0, 2004. Lyon: IarcPress; 2004.

2. Cancer Incidence in Five Continents, Vol VIII, Scientific Publications $\mathrm{N}^{\circ}$ 155, Lyon, IARC, 2002 and Vol. IX IARC Scientific Publications No 160. Lyon: IARC; 2007.

3. Osaka Cancer Registry. Survival of cancer patients in Osaka (1975-89), 1998, Shinohara Publ, Tokyo.

4. Surveillance, Epidemiology, and End Results (SEER), National Cancer Institute, (http://seer.cancer.gov/).

5. Verdecchia A, Francisci S, Brenner H, et al. EUROCARE-4 Working Group. Recent cancer survival in Europe: a 2000-02 period analysis of EUROCARE-4 data. Lancet Oncol. 2007;8:784-96.

6. Cancer Statistics in Japan 2005. National Cancer Center Tokyo, Japan, Tokyo, 2005.

7. Munoz N, Crespi M, Grassi A, et al. Precursor lesions of oesophageal cancer in high-risk populations in Iran and China. Lancet. 1982;1:876-9.

8. He YT, Hou J, Qiao CY. An analysis of esophageal cancer incidence in Cixian county from 1974 to 1996. World J Gastroenterol. 2003;9:209-13.

9. Takubo K, Vieth M, Aryal G, et al. Islands of squamous epithelium and their surrounding mucosa in columnar lined esophagus: a pathognomonic feature of Barrett's esophagus. Human Pathology. 2005;36:269-74.

10. Paris Workshop on Columnar Metaplasia in the Esophagus and the Esophagogastric Junction. Endoscopy. 2005;37:879-920.

11. Vizcaino AP, Moreno V, Lambert R, et al. Time trends incidence of both major histologic types of esophageal carcinomas in selected countries, 1973-1995. Int J Cancer. 2002;99:860-88.

12. Lambert R, Hainaut P, Parkin DM. Premalignant lesions of the esophagogastric mucosa. Semin Oncol. 2004;31:498-512.

13. Lambert R, Hainaut P. Esophageal cancer. II-the precursors. Endoscopy. 2007;39:659-64.

14. Lambert R, Parkin DM. Screening, surveillance \& prevention of gastric cancer. In: Gastrointestinal oncology: principle and practice, Kelsen DH, Daly JM, Kern SE et al, Eds. Lippincott Williams Wilkins Publ. $2^{\text {nd }}$ edition, 2008;231-44.

15. Correa P. Human gastric carcinogenesis: a multistep and multifactorial process. Cancer Res. 1992;52:6735-40.

16. IARC. Monographs on the evaluation of carcinogenic risks to humans, Vol. 61, schistosomes, liver flukes and helicobacter pylori. Lyon: International Agency for Research on Cancer; 1994. p. $177-240$.

17. Jass JR. Classification of colorectal cancer based on correlation of clinical, morphological and molecular features. Histopathology. 2007;50:113-30.

18. The Paris endoscopic classification of superficial neoplastic lesions: esophagus, stomach, and colon. Gastrointest Endosc. 2003;58(Suppl N6):S3-43.

19. Dixon MF. Gastrointestinal epithelial neoplasia: Vienna revisited. Gut. 2002;51:130-1.

20. Kanao H, Tanaka S, Oka S, et al. Narrow-band imaging magnification predicts the histology and invasion depth of colorectal tumors. Gastrointest Endosc. 2009;69(3 Suppl):6316.

21. Kara MA, Peters FP, Rosmolen WD, et al. High-resolution endoscopy plus chromoendoscopy or narrow-band imaging in Barrett's esophagus: a prospective randomized crossover study. Endoscopy. 2005;37:929-36.

22. Kuznetsov K, Lambert R, Rey JF. Narrow-band imaging: potential and limitations. Endoscopy. 2006;38:76-81.

23. Machida H, Sano Y, Yamamoto Y, et al. Narrow-band imaging in the diagnosis of colorectal mucosal lesions: a pilot study. Endoscopy. 2004;36:1094-8.

24. Nakayoshi T, Tajiri H, Matsuda K, et al. Magnifying endoscopy combined with narrow band imaging system for early gastric cancer: correlation of vascular pattern with histopathology. Endoscopy. 2004;36:1080-4.

25. Toshida $\mathrm{T}$, Inoue $\mathrm{H}$, Susui $\mathrm{S}$, et al. Narrow-band imaging system with magnifying endoscopy for superficial esophageal lesions. Gastrointest Endosc. 2004;59:288-95.

26. Gono K, Obi T, Yamaguchi M, et al. Appearance of enhanced tissue features in narrow-band endoscopic imaging. J Biomed Opt. 2004;9:568-77.

27. Endo T, Awakawa T, Takahashi H, et al. Classification of Barrett's epithelium by magnifying endoscopy. Gastrointest Endosc. 2002;55:641-7.

28. Kumagai Y, Monma K, Kawada K. Magnifying chromoendoscopy of the esophagus: in-vivo pathological diagnosis using an endocytoscopy system. Endoscopy. 2004;36:590-4.

29. Kiesslich R, Goetz M, Vieth M, et al. Confocal laser endomicroscopy. Gastrointest Endosc Clin N Am. 2005;15:715-31.

30. Sasajima K, Kudo SE, Inoue H, et al. Real-time in vivo virtual histology of colorectal lesions when using the endocytoscopy system. Gastrointest Endosc. 2006;63:1010-7.

31. Lambert R, Kudo SE, Vieth M, et al. Pragmatic classification of superficial neoplastic colorectal lesions. Gastrointest Endosc. 2009;70:1182-99.

32. Lambert R, Jeannerod M, Rey JF. Eyes wide shut. Endoscopy. 2004;36:723-5.

33. Lambert R, Saito H, Saito Y. High-resolution endoscopy and early digestive cancer. Endoscopy. 2007;39:232-7.

34. Kudo S, Lambert R, Allen JI, et al. Nonpolypoid neoplastic lesions of the colorectal mucosa. Gastrointest Endosc. 2008;68(4 Suppl):S3-47.

35. Inoue H, Rey JF, Lightdale C. Lugol chromoendoscopy for esophageal squamous cell cancer. Endoscopy. 2001;33:75-9.

36. Kiesslich R, Neurath MF. Chromo- and magnifying endoscopy for colorectal lesions. Eur J Gastroenterol Hepatol. 2005;17:793801.

37. Kudo S, Hirota S, Nakajima T, et al. Colorectal tumours and pit pattern. J Clin Pathol. 1994;47:880-5.

38. Nagata S, Tanaka S, Haruma K, et al. Pit pattern diagnosis of early colorectal carcinoma by magnifying colonoscopy: clinical and histological implications. Int J Oncol. 2000;16:927-34. 
39. Tanaka S, Haruma K, Nagata S, et al. Diagnostic of invasion depth in early colorectal carcinoma by pit pattern analysis with magnifying endoscopy. Dig Endosc. 2001;13(Suppl):S2-5.

40. Teixeira CR, Torresini RS, Canali C, et al. Endoscopic classification of the capillary-vessel pattern of colorectal lesions by spectral estimation technology and magnifying zoom imaging. Gastrointest Endosc. 2009;69(3 Suppl):750-6.

41. Sano Y, Saito Y, Fu KI, et al. Efficacy of magnifying endoscopy for the differential diagnosis of colorectal lesions. Dig Endosc. 2005;17:105-16.

42. Kumagai Y, Inoue H, Nagai K, et al. Magnifying endoscopy, stereoscopic microscopy, and the microvascular architecture of superficial esophageal carcinoma. Endoscopy. 2002;34:36975.

43. Yao K, Takaki Y, Ohara J, et al. Magnification endoscopy outlines the microvascular architecture and extent of Barrett's intramucosal carcinoma prior to endoscopic resection. Gastrointest Endosc. 2006;63:1064-5.
44. Yao K, Iwashita A, Kikuchi Y. Novel zoom endoscopy technique for visualizing the microvascular architecture in gastric mucosa. Clin Gastroenterol Hepatol. 2005;3 Suppl 1:S23-6.

45. Wada Y, Kudo SE, Kashida H, et al. Diagnosis of colorectal lesions with the magnifying narrow-band imaging system. Gastrointest Endosc. 2009;70:522-31.

46. Lieberman DA, Weiss DG, Bond JH, et al. Use of colonoscopy to screen asymptomatic adults for colorectal cancer. Veterans Affairs Cooperative Study Group 380. N Engl J Med. 2000;343:162-8.

47. Kaltenbach T, Rouse RV, et al. Prevalence of non-polypoid (flat and depressed) colorectal neoplasms in asymptomatic and symptomatic adults. JAMA. 2008;299:1027-35.

48. Imperiale TF, Glowinski EA, Lin-Cooper C, et al. Five-year risk of colorectal neoplasia after negative screening colonoscopy. N Engl J Med. 2008;359:1218-24.

49. Baxter NN, Goldwasser MA, Paszat LF, et al. Association of colonoscopy and death from colorectal cancer. Ann Intern Med. 2009;150:1-8. 